\title{
Achados histopatológicos de produtos de ressecção em mamaplastias redutoras
}

\author{
Histopathological findings in tissue specimens from reduction mammaplasties
}

\author{
Juliana Régia Furtado \\ Matos $^{1}$ \\ Matheus Martins \\ CAVALCANTE $^{2}$ \\ Sarah Hanna de Carvalho \\ ANDRADE $^{2}$ \\ Salustiano Gomes de \\ PinHO PESSOA ${ }^{3}$
}

Trabalho realizado no Hospital Universitário Walter Cantídio, Universidade Federal do Ceará, Fortaleza, CE, Brasil.

Artigo submetido pelo SGP (Sistema de Gestão de Publicações) da RBCP.

Artigo recebido: $24 / 11 / 2010$ Artigo aceito: 6/2/2011

\begin{abstract}
RESUMO
Introdução: A mamoplastia redutora está entre as cinco cirurgias estéticas mais realizadas no Brasil. Não há consenso na literatura sobre a necessidade do exame de rotina dos tecidos ressecados durante o procedimento. Esse trabalho apresenta a estatística dos espécimes obtidos de mamoplastias redutoras e discute a relevância do detalhamento histopatológico dessas peças. Método: Resultados de 96 peças cirúrgicas de reduções mamárias, realizadas durante o período de 2006 a 2010, foram obtidos de uma única instituição e analisados retrospectivamente. Resultados: Alterações histopatológicas foram observadas em 83,3\% dos espécimes. As lesões não-proliferativas benignas foram os principais achados, sendo fibrose, adenose e cistos as mais frequentes. Alterações proliferativas foram encontradas em percentual significativo das pacientes (13,54\%). Uma paciente $(1,16 \%)$, com 50 anos de idade, apresentou carcinoma lobular in situ. Pacientes na faixa etária menor que 40 anos não apresentaram lesões malignas ou pré-malignas. Conclusões: A prática rotineira de rastreamento histopatológico de peças de mamoplastias redutoras em pacientes jovens parece discutível, embora tenha sido benéfica em uma paciente com idade superior a 40 anos. Trabalhos adicionais são necessários para definir um grupo em que medidas preventivas teriam melhor relação custo-benefício.
\end{abstract}

Descritores: Mamoplastia. Neoplasias da Mama. Histologia.

\begin{abstract}
Background: Reduction mammaplasty ranks among the top five aesthetic surgical procedures performed in Brazil. No standard pathology assessment for reduction mammaplasty exists. This study presents the reduction mammaplasty specimens findings statistic, and a review about the real relevance of this exam. Methods: Findings of 96 reduction mammaplasty specimens over a 5 years period at a single institution were retrospectively examined. Results: Pathologic findings were present in $83.3 \%$. Non proliferative benign findings are the most frequent lesions, such as fibrosis, adenosis and cists. Proliferative findings are present in $13.54 \%$. A fifth years old patient (1.16\%) presented lobular carcinoma in situ. Patients younger than 40 years old did not present maligns and pre-maligns lesions. Conclusions: Routinely practice of histopathological analysis of reduction mammaplasty in young people seems arguable. But this routine was benefit for a patient older than 40 years old. Others studies are need to define a group where preventive measures have a better cost-benefit.
\end{abstract}

Keywords: Mammaplasty. Breast Neoplasms. Histology.

1. Médica Residente do Serviço de Cirurgia Plástica e Microcirurgia Reconstrutiva do Hospital Universitário Walter Cantídio, Membro Aspirante da Sociedade Brasileira de Cirurgia Plástica (SBCP), Fortaleza, CE, Brasil.

2. Acadêmico de Medicina da Universidade Federal do Ceará, Fortaleza, CE, Brasil.

3. Cirurgião plástico, Membro Titular da SBCP; Regente do Serviço de Cirurgia Plástica e Microcirurgia Reconstrutiva do Hospital Universitário Walter Cantídio da Universidade Federal do Ceará, São Lucas - Hospital de Cirurgia e Anestesia, Fortaleza, CE, Brasil. 


\section{INTRODUÇÃO}

A mamoplastia redutora está entre as cinco cirurgias estéticas mais realizadas no Brasil. Estima-se que, em 2008, tenham sido realizadas aproximadamente 55.000 reduções de mama no país ${ }^{1}$. A principal indicação cirúrgica é o tratamento da macromastia, caracterizada pelo aumento do estroma mamário relacionado a fatores genéticos e hormonais ${ }^{2,3}$.

A redução da mama tem como objetivo a obtenção do equilíbrio estético e postural em pacientes com macromastia, assimetria mamária congênita ou simetrização após mastectomias contralaterais ${ }^{3}$.

As candidatas a mamoplastia redutora são submetidas a um rastreamento pré-operatório para detecção de alterações potencialmente neoplásicas ${ }^{3}$. Apesar das pacientes submetidas a rastreamento pré-operatório negativo estarem sob baixo risco de apresentar carcinoma mamário oculto, há décadas tem sido uma prática comum a análise histológica dos tecidos ressecados ${ }^{4}$.

Há infindáveis discussões na literatura sobre a necessidade do exame de rotina dos tecidos ressecados durante o procedimento cirúrgico. A incidência extremamente baixa de alterações neoplásicas nesses espécimes e a faixa etária jovem das pacientes submetidas a essas cirurgias são fatores que devem ser considerados quando se questiona a relação custo-benefício dessa prática ${ }^{4}$.

Por outro lado, cerca de $60 \%$ das pacientes sem alterações nos exames pré-operatórios apresentam alterações benignas nas peças cirúrgicas de mamoplastias. A análise dos tecidos ressecados poderia diagnosticar lesões proliferativas pré-malignas e possibilitar o seguimento adequado dessas pacientes ${ }^{5}$.

Assim, estudos regionais devem ser realizados para traçar o perfil de incidência das principais alterações encontradas na população e determinar a real importância da análise das peças ressecadas em termos de saúde pública.

O presente trabalho apresenta a estatística dos espécimes obtidos de pacientes submetidas à mamaplastia redutora, nos últimos 5 anos, em nosso Serviço, e discute alguns aspectos relevantes sobre o real benefício do detalhamento histopatológico dessas peças, por meio de revisão da literatura.

\section{MÉTODO}

Trata-se de estudo de abordagem quantitativa, descritiva e retrospectiva, realizado no Serviço de Cirurgia Plástica e Microcirurgia do Hospital Universitário Walter Cantídio.

Resultados de 96 peças cirúrgicas originadas de 50 mamoplastias redutoras estéticas, realizadas durante o período de 2006 a 2010, foram obtidos dos laudos histopatológicos arquivados nos prontuários e analisados.
Todas as pacientes estudadas foram submetidas a rastreamento pré-operatório com ultrassonografia mamária e mamografia. Nenhuma paciente da amostra apresentou lesões suspeitas de malignidade nos exames pré-operatórios.

As lesões benignas não-neoplásicas foram analisadas individualmente. As lesões proliferativas intraductais foram classificadas em hiperplasia ductal usual (HDU), hiperplasia ductal atípica (HDA) e carcinoma ductal in situ (CDIS), segundo critérios da Organização Mundial de Saúde (2003) ${ }^{3}$. A hiperplasia lobular atípica e o carcinoma lobular in situ foram computados separadamente.

Os dados foram analisados pelo programa Statistical Package for Social Science (SPSS) versão 16.0.

\section{RESULTADOS}

Foram estudadas 96 peças cirúrgicas provenientes de 50 reduções de mama bilaterais. Quatro pacientes possuíam laudo histopatológico apenas de uma mama.

A média etária das pacientes submetidas a mamoplastia redutora foi 31,88 anos, variando de 15 a 57 anos de idade $(\mathrm{sd}=11,45)$.

Alterações histopatológicas foram observadas em $83,3 \%$ dos espécimes analisados. As lesões não-proliferativas benignas foram os principais achados, sendo fibrose $(73,95 \%)$, adenose $(52,08 \%)$ e cistos $(43,75 \%)$ as mais frequentes (Tabela 1).

Alterações proliferativas foram encontradas em percentual significativo das pacientes $(13,54 \%)$. O papiloma intraductal e a cicatriz radial ocorreram em $3,12 \%$ dos espécimes cada. A hiperplasia ductal usual foi encontrada em $10,41 \%$ das peças, sendo $50 \%$ delas com idade inferior 35 anos. Hiperplasia ductal usual e carcinoma ductal in situ não ocorreram nas amostras estudadas. Uma (1,16\%) paciente, com 50 anos de idade, apresentou carcinoma lobular in situ no laudo histológico e, após avaliação do Serviço de Mastologia, optou-se pela conduta não-cirúrgica e seguimento adequado (Tabela 2).

Tabela 1 - Incidência de alterações histopatológicas não-proliferativas nas peças cirúrgicas.

\begin{tabular}{l|c|c}
\hline \multicolumn{1}{c|}{ Alteração histopatológica } & $\mathbf{N}$ & \% \\
\hline Fibrose & 71 & $73,95 \%$ \\
\hline Adenose & 50 & $52,08 \%$ \\
\hline Cisto & 42 & $43,75 \%$ \\
\hline Hiperplasia fibroadenomatosa & 17 & $17,70 \%$ \\
\hline Metaplasia apócrina & 9 & $9,37 \%$ \\
\hline Microcalcificações & 6 & $6,25 \%$ \\
\hline Hiperplasia pseudoangiomatosa & 3 & $3,12 \%$ \\
\hline Fibroadenoma & 2 & $2,08 \%$ \\
\hline
\end{tabular}


Tabela 2 - Incidência de alterações histopatológicas proliferativas nas peças cirúrgicas.

\begin{tabular}{l|c|c}
\hline \multicolumn{1}{c|}{ Alteração histopatológica } & N & $\mathbf{\%}$ \\
\hline Papiloma intraductal & 3 & $3,12 \%$ \\
\hline Cicatriz radial & 3 & $3,12 \%$ \\
\hline Hiperplasia ductal usual & 10 & $10,41 \%$ \\
\hline Hiperplasia ductal atípica & - & 0 \\
\hline Carcinoma ductal in situ & - & 0 \\
\hline Carcinoma lobular in situ & 1 & $1,04 \%$ \\
\hline
\end{tabular}

\section{DISCUSSÃO}

Vários trabalhos têm demonstrado a ocorrência de alterações histológicas nos espécimes cirúrgicos provenientes de mamaplastia redutora. Também tem sido questionada a importância do detalhamento microscópico das peças cirúrgicas ${ }^{6}$.

Uma pesquisa realizada com médicos britânicos, em 1994, demonstrou que $89 \%$ dos cirurgiões enviam as peças ressecadas para biopsia rotineiramente e $42 \%$ afirmaram que encontraram pelo menos um caso de carcinoma de mama nos tecidos ressecados ${ }^{4}$.

Em diferentes séries, o carcinoma oculto foi encontrado em 0,05 a 1,66\% dos espécimes de redução mamária e o risco variou de acordo com a precisão dos métodos diagnósticos pré e pós-operatórios ${ }^{4}$.

Snyderman e Lizardo 7 , em uma série com 5008 espécimes, reportaram deteç̧ão de câncer em $0,4 \%(0,24-0,62 \%)$, nesse estudo foram incluídos pacientes com carcinoma detectado já no pré-operatório. Jansen et al. ${ }^{8}$, em um estudo similar, encontraram incidência de $0,16 \%(0,04-0,4 \%)$ de neoplasia em 2.576 peças. Recentemente, Tang et al. ${ }^{9}$ documentaram carcinoma invasivo em $0,06 \%(0,03-0,09 \%)$, em 27.500 reduções mamárias, mas não incluíram carcinoma ductal in situ nos resultados. Outros autores descreveram com detalhes os achados patológicos nas peças cirúrgicas, mas não detectaram câncer nesses estudos ${ }^{4}$.

Esses estudos refletem a diminuição na detecção de câncer oculto nos espécimes de mamaplastia redutora em anos recentes, que tem sido explicada por avanços tecnológicos nos métodos de detecção precoce, progressos na educação populacional, grupo mais jovem de pacientes realizando cirurgia mamária e rastreamento pré-operatório mais completo ${ }^{4}$.

A amostra estudada apresenta incidência de carcinoma oculto de $1,04 \%$ das peças cirúrgicas, superior aos principais estudos citados, mas compatível com algumas séries da literatura. $\mathrm{Na}$ análise apenas da população com idade superior a 40 anos, a incidência de neoplasia oculta representou 8,33\% dos espécimes ressecados e nenhuma paciente abaixo dessa faixa etária apresentou carcinoma nas peças examinadas. Ressalta-se que a lesão diagnosticada não é invasiva e alguns trabalhos não a consideram nas casuísticas apresentadas.

Atualmente não é recomendado o rastreamento para câncer de mama por qualquer modalidade, exceto o exame clínico, em mulheres com idade inferior a 35 anos, mas pacientes jovens são submetidas a rastreamento histopatológico após realização de cirurgia estética de mama sem evidência científica clara. Há relatos na literatura de pacientes que tiveram achados de importância clínica incerta e que foram submetidas a anos de ansiedade e múltiplos procedimentos cirúrgicos sem benefício comprovado ${ }^{4}$.

Alguns autores revelaram que parte da dificuldade do manejo das pacientes com alterações histopatológicas surge porque os espécimes não são orientados durante a cirurgia, não sendo possível determinar com precisão a área afetada. A técnica de ressecção que inclui a mobilização de tecidos distorce a arquitetura mamária no pós-operatório. Assim, a avaliação radiológica subsequente não é simples e o manejo cirúrgico é um desafio ao mastologista. Embora cânceres pós-redução mamária tenham sido encontrados em estádios mais precoces, essas pacientes foram mais propensas a serem submetidas à mastectomia que à cirurgia conservadora ${ }^{4}$. Assim, torna-se evidente que o rastreamento pré-operatório criterioso é imprescindível durante o preparo para a cirurgia de mama.

Nesse trabalho, uma paciente apresentava carcinoma lobular in situ e não necessitou de terapêutica cirúrgica, sendo o seguimento rigoroso e frequente a conduta indicada. Assim, o resultado beneficiou a paciente em questão e alertou-a para o rastreamento adequado nos anos subsequentes.

Seguindo outra linha de análise, de acordo com a literatura, cerca de $60 \%$ das pacientes sem alterações pré-operatórias apresentam alterações benignas nas peças cirúrgicas de mamoplastias 5 .

Tafuri e $\mathrm{Gobi}^{3}$, em um estudo realizado no Brasil, revisaram 867 peças originadas de mamoplastia redutora bilateral, em pacientes assintomáticas, e verificaram que 50,9\% apresentavam alterações fibrocísticas e 10,1\%, hiperplasias epiteliais, lesões proliferativas pré-malignas da mama que requerem seguimento mais amiúde. Esses dados são semelhantes aos publicados por outros autores.

A avaliação das lesões proliferativas nas peças estudadas pode individualizar pacientes e definir estratégias de seguimento ou medidas preventivas, como uso de antiestrogênicos e mastectomia profilática ${ }^{3}$.

A casuística em questão demonstrou $83,3 \%$ de alterações histológicas, sendo principalmente lesões benignas não-proliferativas. Ressalva-se que nessa estatística não foram excluídas as peças que apresentavam alterações benignas nos exames de imagem pré-operatórios. Lesões proliferativas, como o papiloma intraductal e a cicatriz 
radial, ocorreram em $3,12 \%$ dos casos cada. A hiperplasia ductal usual foi observada em $10,41 \%$ dos espécimes analisados. Lesões atípicas e pré-malígnas não foram encontradas.

Alguns estudos tentaram individualizar as pacientes em grupos de maior risco para desenvolvimento de carcinoma mamário pós-cirurgia redutora e demonstraram incidência superior nos tecidos ressecados.

Em outro trabalho brasileiro, Souza et al. ${ }^{6}$ encontraram alterações histopatológicas em $98,63 \%$ das 1018 peças analisadas e distribuíram-nas por faixa etária. Também relataram 3 achados de lesões pré-malígnas da mama, uma delas ocorrendo em paciente com idade inferior a 35 anos $(0,25 \%$ de 406 pacientes do grupo). Nenhum achado de carcinoma foi relatado nesse estudo.

Um trabalho sueco, que também analisou os resultados por faixa etária, encontrou incidência de $8 \%$ de carcinoma in situ nas peças provenientes de mamoplastia redutora em pacientes com idade superior a 40 anos ${ }^{10}$.

Um estudo prospectivo recente também apresentou taxa significativamente maior à descrita na literatura. As hiperplasias atípicas e os carcinomas foram encontrados em 12,4\% e apenas em pacientes com idade superior a 40 anos $(6,2 \%$ em pacientes com idade superior a 40 anos e $7,9 \%$ acima de 50 anos $)^{11}$.

Cowell et al. ${ }^{2}$ relataram que a incidência de câncer nas peças poderia variar com a indicação cirúrgica e estratificaram os dados em cirurgias para macromastia, assimetria congênita e redução contralateral para simetrização pósmastectomia. Em 800 reduções (611 bilaterais e 189 unilaterais), encontrou-se $0,8 \%$ câncer $(0,28-1,63 \%)$, sendo $0,7 \%$ no grupo da macromastia $(0,18-1,68 \%), 0 \%$ no da assimetria mamária e 1,2\% no da reconstrução para simetrização $(0,14$ $4,20 \%$ ). O grupo de redução mamária contralateral para reconstrução apresentou risco maior que a população normal para neoplasia. Ressalta-se que o câncer de mama per si já é um fator de risco importante para neoplasia contralateral.

Assim, de acordo com a literatura, nas pacientes com idade superior a 40 anos ou submetidas à redução mamária para simetrização pós-mastectomia, o exame histológico das peças cirúrgicas poderia encontrar reforço científico. Nesse estudo, não avaliamos pacientes do grupo da reconstrução contralateral.

\section{CONCLUSÕES}

A casuística apresentada demonstra que a incidência de carcinoma oculto na instituição estudada é semelhante à relatada na literatura. Pacientes na faixa etária inferior a 40 anos não apresentaram câncer ou lesões pré-malignas nos espécimes estudados. Assim, a prática rotineira de rastreamento histopatológico de peças ressecadas de mamoplastias redutoras em pacientes jovens parece discutível, embora tenha sido benéfica em uma paciente com idade superior a 40 anos. Trabalhos adicionais são necessários para definir um grupo em que medidas preventivas teriam uma melhor relação custo-benefício.

\section{REFERÊNCIAS}

1. Pesquisa Datafolha encomendada para Sociedade Brasileira de Cirurgia Plástica. Disponível em: <http://www2.cirurgiaplastica.org. br/index.php?option=com_content \&view=article\&id=100:plasticade-mama-ultrapassa-lipo $\&$ catid $=42$ : saiu-na-midia $\&$ Ite $\mathrm{mid}=87>$. Acesso em: 15 jan 2011.

2. Cowell AS, Kukreja J, Breuing KH, Lester S, Orgill DP. Occult breast carcinoma in reduction mammaplasty specimens: 14-year experience. Plast Reconstr Surg. 2004;113(7):1984-8.

3. Tafuri LSA, Gobbi H. Hiperplasias epiteliais em espécimes de mamoplastia redutora estética bilateral e mamoplastia redutora contralateral a câncer de mama. J Bras Patol Med Lab. 2005;41(2):135-41.

4. Keshtgar M, Hamidian Jahromi A, Davidson T, Escobar P, Mallucci $\mathrm{P}$, Mosahebi A, et al. Tissue screening after breast reduction. BMJ. 2009;338:b630.

5. Ayhan S, Basterzi Y, Yavuzer R, Latifoglu O, Cenetoglu S, Atabay K, et al. Histologic profiles of breast reduction specimens. Aesthetic Plast Surg. 2002;26(3):203-5.

6. Souza LHG, Oliveira Segundo SWG, Dalul DB, Morais CM, Antunes DE, Pirani F, et al. Estudo histopatológico de 1018 peças cirúrgicas de mamaplastia redutora. Rev Bras Cir Plást. 2005;20(3):173-5.

7. Snyderman RK, Lizardo JG. Statistical study of malignancies found before, during, or after routine breast plastic operations. Plast Reconstr Surg. 1989;25:253-6.

8. Jansen DA, Murphy M, Kind GM, Sands K. Breast cancer in reduction mammaplasty: case report and a survey of plastic surgeons. Plast Reconstr Surg. 1998;101(2):361-4.

9. Tang CL, Brown MH, Levine R, Sloan M, Chong N, Holowaty E. Breast cancer found at the time of breast reduction. Plast Reconstr Surg. 1999;103(6):1682-6.

10. Bondeson L, Linell F, Ringberg A. Breast reductions: what to do with all the tissue specimens? Histopathology. 1985;9(3):281-5.

11. Ambaye AB, MacLennan SE, Goodwin AJ, Suppan T, Naud S, Weaver DL. Carcinoma and atypical hyperplasia in reduction mammaplasty: increased sampling leads to increased detection. A prospective study. Plast Reconstr Surg. 2009;124(5):1386-92.

\section{Correspondência para: Juliana Régia Furtado Matos}

Rua Mombaça, 164 - Aldeota - Fortaleza, CE, Brasil - CEP: 60160-190

E-mail: julianarfm@gmail.com 